\title{
Author Index to Abstract Parts 1-3
}

Numbers in bold type refer to the abstract part; numbers in regular type refer to abstract numbers Albignac, R. 1/1 Agetsuma, N. 3/7 Anderson, J.R. 1/21 Araujo, A. 1/2 Arruda, M.F. 1/2

Barbe, S. 1/3 Bellec,S. 1/9 Berge, C. 1/4 Bescond,P. 1/12 Bibollet-Ruche, F. 1/13 Braga, J. 1/6 Brandibas, P. 1/7 Boiler, F. 1/11 Bons, N. 1/5, 1/17, 1/19, 1/31,1/36 Borries, C. 3/13 Bowman, J.E. 2/4 Byrne, R.W. 3/1

Carval, N. 1/12 Casinos, A. 1/4 Chalmeau, R. 1/7, 1/8 Chamberlain, A. 3/11 Cornet, M. 1/13 Dabringhaus, A. 1/16 Demaria-Pesce, V. 1/24 Deputte, B.L. 1/3, 1/9 Depy, D. 1/10 Dhenain, M. 1/11 Dixson, A.F. 2/1 Doumergue, C. 1/5 Dunbar, R.I.M. 2/2 Durand, J.P. 1/13

Elkin,L. 1/15

Fagot, J. 1/10,1/37 Fradin, Y. 1/12

Galat, G. 1/13 Galat-Luong, A. 1/13 Gallo, A. 1/7, 1/8 Gauquelin-Koch, G. 1/24 Gauthier, C.-A. $1 / 14$

Gautier,J.P. 1/15 Gilissen, E. 1/16 Guiraud, I. 1/17

Hachem, R. 1/18

Hall, L.M. 3/2

Ham, R. 3/3

Hardie, S.H. 3/4

Henzi, S.P. $3 / 6$

Hill, D.A. 3/7,3/8,3/10

Honess, RE. $3 / 5$

Jallageas, V. 1/19, 1/36 Jones, D.S. 3/2 Jouanjean, A. 1/9 Jouffroy, F.K. 1/4

Keverne, E.B. 2/3 Koenig, A. 3/13

Leclercq, B. 1/20 Lee, P.C. 2/4 Lucas, P. 3/8 Ludes,E. 1/21, 1/27 Lycett, J.E. 3/6

MacLarnon, A.M. 2/5 Majoral, M. 1/4 Malecki, H. 1/22, 1/23 Martin, F. 1/24 Martin, M.-P. 1/25

Ménard,N. 1/18 Mestre, N. 1/5, 1/36 Michot,J.L. 1/11 Montagnon, D. 1/27 Muroyama, Y. 1/26

Neveu, H. 1/27 Nicolaidis, S. 1/24

Papin,T. 1/14 Perret, M. 1/28, 1/29 Petter, A. 1/5 Pichon, G. 1/13 Picq,J.L. 1/11,1/30 Pierre, G $1 / 9$

Privat, N. 1/31 Ponsard, S. 1/1 Pourrut,X. 1/13

Rajpurohit, L.S. 1/32 Rosolen, S.G 1/22 Ross, C. 2/5 Rumpler, Y. 1/27

Sambrook, T. 3/9 Santini-Palka, M. 1/14, 1/20 Schilling, A. 1/33, 1/34 Schlaug, G 1/16

Schormann, T. 1/16 Sellers, W.I. 2/6 Senut, B. 1/35 Servière, J. 1/34 Silhol,S. 1/36 Sprague, D.A. $3 / 10$ Suzuki, S. 3/10

Thi Minh Tarn, H. 1/23 Thorpe, S.K. 3/11

Vauclair, J. 1/10, 1/37 Vandermecken, C. 1/22 Van Hung, T. 1/23 Vincent, J.-C. 1/12

Visalberghi, E. 1/8 Volk,A. 1/11 Voysey, B. 3/12 Vrot, M. 1/9

Wheeler, P. 2/7 Whiten, A. 3/9 Winkler, P. 3/13 Wood, B.A. 3/2

Yamagiwa, J. 3/14 Yioulatos, D. 1/38

Zilles,K. 1/16 